\title{
THE FIX-POINTS AND FACTORIZATION OF MEROMORPHIC FUNCTIONS
}

\author{
BY \\ FRED GROSS( ${ }^{1}$ ) AND CHUNG-CHUN YANG
}

\begin{abstract}
In this paper, we use the Nevanlinna theory of meromorphic functions and a result of Goldstein to generalize some known results in factorization and fixpoints of entire functions. Specifically, we prove

(1) If $f$ and $g$ are nonlinear entire functions such that $f(g)$ is transcendental and of finite order, then $f(g)$ has infinitely many fix-points.

(2) If $f$ is a polynomial of degree $\geqq 3$, and $g$ is an arbitrary transcendental meromorphic function, then $f(g)$ must have infinitely many fix-points.

(3) Let $p(z), q(z)$ be any nonconstant polynomials, at least one of which is not $c$-even, and let $a$ and $b$ be any constants with $a$ or $b \neq 0$.

Then $h(z)=q(z) \exp \left(a z^{2}+b z\right)+p(z)$ is prime.
\end{abstract}

I. Introduction. A meromorphic function $h(z)=f(g(z))$ is said to have $f(z)$ and $g(z)$ as left and right factors respectively, provided that $f(z)$ is meromorphic and $g(z)$ is entire ( $g$ may be meromorphic when $f$ is rational). Such a composition $f(g)$ is called a factorization of $h . h(z)$ is said to be prime (pseudo prime) if every factorization of the above form implies that one of the functions $f$ or $g$ is bilinear (a polynomial or $f(z)$ is rational). $h(z)$ is said to be $E$-prime ( $E$-pseudo prime) if every factorization of the above form into entire factors implies that one of the functions $f$ or $g$ is linear (a polynomial).

Let $f(z)$ be a nonconstant meromorphic function in the whole finite plane. A point $z_{0}$ is a fix-point of $f$ if and only if $f\left(z_{0}\right)=z_{0}$. In 1926 Fatou [4] announced that when $f$ is entire, then $f(f)$ has infinitely many fix-points unless $f$ is a polynomial. This result was proved and generalized by Rosenbloom [12] as follows:

THEOREM A. If $f$ and $g$ are transcendental entire functions, then either $f$ or $f(g)$ has infinitely many fix-points.

In the same paper, Rosenbloom also showed

THEOREM B. Let $p(z)$ be a polynomial of degree $k \geqq 2$, and $g(z)$ be transcendental entire, then $p(g)$ has infinitely many fix-points.

Received by the editors May 20, 1971.

AMS 1969 subject classifications. Primary 3055, 3060, 3061; Secondary 3930.

$K e y$ words and phrases. Entire functions, meromorphic functions, prime functions, $E$ pseudo prime, $E$-prime, fix-points, factorization, differential polynomials, zeros, poles, Nevanlinna theory of meromorphic functions, $c$-even polynomials.

(1) The research of the first author was partially supported by the National Science Foundation under Grant GS-13875.

Copyright (C) 1972, American Mathematical Society 
The first author proved the following generalization of Theorem A.

THEOREM C [6]. Let $F\left(F^{*}\right)$ denote the family of entire (meromorphic) functions with at most a finite number of fix-points. Then (i) every entire function has at most one factorization $f(g(z)), f$ transcendental, $f \in F, g$ entire; (ii) every meromorphic function has at most two distinct factorizations $f_{i}\left(g_{i}\right), f_{i}$ meromorphic, $f_{i}$ not rational, $f_{i} \in F^{*}, g_{i}$ entire.

In particular, if $f$ is transcendental meromorphic and $g$ and $h$ are transcendental entire then one of $f(z), f(g(z)), f(g(h(z)))$ has infinitely many fix-points.

It was conjectured in $[1$, p. 542] that if $f$ is transcendental entire and $g$ is nonlinear entire, then $f(g)$ has infinitely many fix-points. The conjecture was also made when $f$ is meromorphic [5]. However, nothing was stated about the case when $f$ is rational and $g$ meromorphic.

The purpose of this note is to generalize Theorem B to meromorphic functions and to point out that a recent result of Goldstein [10] yields a partial answer to the above conjecture. We shall also obtain some interesting results on the factorization of entire functions. Specifically, we shall prove

THEOREM 1. Let $f$ and $g$ be entire and nonlinear and such that $F=f(g)$ is transcendental and of finite order, then $F$ has infinitely many fix-points.

As a generalization of Theorem $B$, we prove

THEOREM 2. Let $p(z)$ be a polynomial of degree $n \geqq 3$, and let $f$ be transcendental meromorphic, then $p(f)$ has infinitely many fix-points.

When $n=2$, we prove

THEOREM 3. Let $p(z)=z^{2}, f$ transcendental meromorphic, then $p(f)$ has only finitely many fix-points if and only if

$$
f(z)=\frac{Q\left(z^{1 / 2}\right) \exp \left(\gamma\left(z^{1 / 2}\right)\right)+Q\left(-z^{1 / 2}\right) \exp \left(\gamma\left(-z^{1 / 2}\right)\right)}{\left(Q\left(z^{1 / 2}\right) / z^{1 / 2}\right) \exp \left(\gamma\left(z^{1 / 2}\right)\right)-\left(Q\left(-z^{1 / 2}\right) / z^{1 / 2}\right) \exp \left(\gamma\left(-z^{1 / 2}\right)\right)},
$$

where $Q(z)$ is a polynomial, and $\gamma(z)$ is entire.

REMARK. When $p(z)$ is an arbitrary nonlinear polynomial quadratic form, a similar result holds.

Definition 1. Let $c$ be any complex number. A polynomial $p(z)$ is said to be $c$-even if and only if $p(z)=p(-z-2 c)$.

THEOREM 4. Let $p(z)$ and $q(z)$ be any nonconstant polynomials, at least one of which is not c-even, and let $a$ and $b$ be any constants (at least one of them different from zero). Then $h(z)=q(z) \exp \left(a z^{2}+b z\right)+p(z)$ is prime.

In particular, we have

Corollary. Let $P(z), Q(z)$ be two polynomials, $Q(z) \not \equiv 0, P(z) \not \equiv$ constant. Then $Q(z) e^{z}+P(z)$ is prime. 
This extends an earlier result of the first author and Baker [1, p. 34]. We remark here that by using arguments similar to the proof of Theorem 4, one can obtain the following two results.

THEOREM 5. Let $h_{1}$ be a nonzero polynomial, and $h_{2}$ (非onstant) be an entire function of order less than the degree of the polynomial $p(z)$. Then the only possible factorization of $h_{1}(z) e^{p(z)}+h_{2}(z)$ is of the form $h_{1}(z) e^{p(z)}+h_{2}(z)=f(Q(z))$, where $Q(z)$ is a polynomial of degree no greater than the degree of $p(z)$.

THEOREM 6. Let $h_{1}(z)$ be a nonzero polynomial, and $h_{2}(z)$ (隹constant) be an entire function of order less than one. Then $h_{1}(z) e^{z}+h_{2}(z)$ is prime.

REMARK. $h_{1}, h_{2}$ can be assumed to have a finite number of poles. It follows that $Q$ and $P$ in the corollary of Theorem 4 can be replaced by rational functions.

The tool we employ here is Nevanlinna's theory of meromorphic functions but the approach will be entirely different from Rosenbloom's. It is assumed that the reader is familiar with the fundamental concept of Nevanlinna's first and second fundamental theorems as well as with its usual symbols: $N(r, f), T(r, f), \delta(r, f)$, etc.

II. Preliminaries. We shall need the following lemmas.

First, we state the following definition:

Definition 2. A differential polynomial in $f$ means a polynomial in $f$ and its derivatives with the coefficients $b(z)$ meromorphic and satisfying $T(r, b(z))=S(r, f)$, where $S(r, f)$ denotes any quantity satisfying $S(r, f)=o\{T(r, f)\}$ for all $r$ outside a set of finite measure.

Lemma 1 (Tumura-Clunie [9, p. 69]). Suppose that $f(z)$ is meromorphic and not constant in the plane, that $g(z)=f^{n}(z)+p_{n-1}(f)$, where $p_{n-1}(f)$ is a differential polynomial in $f$ of degree at most $n-1$, and that $N(r, f)+N(r, 1 / g)=S(r, f)$. Then $g(z)=h^{n}(z), h(z)=f(z)+(1 / n) a(z)$ and $h^{n-1}(z) a(z)$ is obtained by substituting $h(z)$ for $f(z), h^{\prime}(z)$ for $f^{\prime}(z)$, etc., in the terms of degree $n-1$ in $p_{n-1}(f)$.

Regarding this lemma we would like to remark the following:

(i) If $P_{n-1}(f)=a_{0}(z) f^{n-1}+$ terms of degree $n-2$ at most, then $h^{n-1}(z) a(z)$ $=a_{0}(z) h^{n-1}$, therefore $a(z)=a_{0}(z)$, and in this case we have $g(z)=\left(f(z)+\left(a_{0}(z) / n\right)\right)^{n}$.

(ii) If $F$ is transcendental with a finite number of poles, then the following identity,

$$
F^{n}(z)+a_{1}(z) F^{n-1}(z)+\cdots+a_{n}(z) \equiv 0,
$$

holds $\left(a_{i}(z)\right.$ are rational functions) if and only if $a_{i}(z) \equiv 0$ for $i=1,2, \ldots, n$.

Our next result follows from a proof of Goldstein [10].

LEMma 2. Let $p(z)$ be a polynomial of degree $k \geqq 1, h_{1}$ be a nonzero polynomial, and $h_{2}(\not \equiv 0)$ be an entire function of order less than $k$. Then $F \equiv h_{1}(z) e^{p(z)}+h_{2}(z)$ is E-pseudo prime. 
Proof. Suppose $F=f(g)$ with $f, g$ transcendental entire. We rewrite

$$
F \equiv h_{1}(z) e^{p(z)}+h_{2}(z)=h_{2}(z)\left(\left(h_{1}(z) / h_{2}(z)\right) e^{p(z)}+1\right) .
$$

Then $H(z)=\left(h_{1}(z) / h_{2}(z)\right) e^{p(z)}+1$ is a meromorphic function of regular growth of order $k$ with $\delta(-1, H)=1$. Then the estimate [2, p. 280] holds for $H$ and since $h_{2}(z)$ is entire with order less than $k$, the estimate holds when $H$ is replaced by $F$ and $-\pi / 16$ replaced by $-\pi / 16+\varepsilon$ for some constant $\varepsilon>0$ but less than $\pi / 16$. The remainder of the proof will be exactly the same as Goldstein's so we omit the details here.

LEMMA 3 [7]. Let $f$ be entire and nonperiodic, then $f$ is prime if and only if $f$ is E-prime.

REMARK. It is easy to verify that all the functions we have considered in Theorems 4, 5, 6 are nonperiodic entire functions. Therefore, according to Lemma 3, we shall only need to consider factorizations with entire factors.

LEMMA 4 (BOREL [2]). Let $a_{i}(z)$ be an entire function of order $\rho$, let $g_{i}(z)$ also be entire and let $g_{j}(z)-g_{i}(z)(i \neq j)$ be a transcendental function or polynomial of degree higher than $\rho$, then

$$
\sum_{i=1}^{n} a_{i}(z) \exp \left(g_{i}(z)\right)=a_{0}(z)
$$

holds only when $a_{0}(z)=a_{1}(z)=\cdots=a_{n}(z)=0$.

REMARK. This lemma was first stated by Borel, but the first complete proof was given by Nevanlinna [11].

III. Proofs of the main results.

3.1. Proof of Theorem 2. Suppose the theorem is false, i.e. $p(f)$ has only finitely many fix-points. Then according to Lemma $1, f(p)$ also has only finitely many fix-points.

Let us first observe that if $f$ and $g$ are entire, or $f$ is meromorphic and $g$ is a polynomial, then $f(g)$ has infinitely many fix-points if and only if $g(f)$ has infinitely many fix-points. For let $z_{0}$ be a fix-point of $f(g)$. Then $f\left(g\left(z_{0}\right)\right)=z_{0}$, and hence $g\left(f\left(g\left(z_{0}\right)\right)\right)=g\left(z_{0}\right)$. This shows that $g\left(z_{0}\right)$ is a fix-point of $g(f)$. Now let $z_{1}, z_{2}$ be any two distinct fix-points of $f(g)$. If $g\left(z_{1}\right)=g\left(z_{2}\right)$ then $z_{2}=f\left(g\left(z_{2}\right)\right)=f\left(g\left(z_{1}\right)\right)=z_{1}$ a contradiction. Hence, we have a one-to-one map from the set of fix-points of $f(g)$ into the set of fix-points of $g(f)$ and vice versa.

Let

$$
f(z)=f_{1}(z) / f_{2}(z)
$$

where $f_{1}$ and $f_{2}$ are entire functions. Then it is easy to verify from this and the above observation that $f_{1}[p(z)]-z f_{2}[p(z)]$ and $f_{2}^{n}(z) p\left(f_{1} / f_{2}\right)-z f_{2}^{n}(z)$ are entire functions with a finite number of zeros. Consequently,

$$
f_{1}[p(z)]-z f_{2}[p(z)]=Q(z) e^{\alpha(z)}
$$


where $Q$ is a polynomial and $\alpha$ is an entire function, and

$$
f_{2}^{n}(z) p\left(f_{1} / f_{2}\right)-z f_{2}^{n}(z)=R_{0}(z) e^{\beta(z)},
$$

where $R_{0}$ is a polynomial and $\beta$ is an entire function.

Replace $z$ by $p(z)$ in (3), use (2) to obtain

$$
f_{2}^{n}(p(z)) p\left\{z+\left(Q e^{\alpha} / f_{2}(p)\right)\right\}-p(z) f_{2}^{n}(p)=s(z) e^{\gamma(z)},
$$

where $s$ is a polynomial and $\gamma$ is an entire function.

By Taylor's Theorem, we have

(5) $p^{\prime}(z) Q e^{\alpha} f_{2}^{n-1}(p)+\frac{1}{2} p^{\prime \prime}(z)\left(Q e^{\alpha}\right)^{2} f_{2}^{n-2}(p)+\cdots+(1 / n !) p^{(n)}(z)\left(Q e^{\alpha}\right)^{n}=s e^{\gamma}$.

The left-hand side of (5) is a homogeneous polynomial in $f_{2}(p)$ and $Q e^{\alpha}$ with degree $n-1$.

Dividing $\left(Q e^{\alpha}\right)^{n}$ on both sides of (5) and setting

$$
F(z)=f_{2}(p) / Q e^{\alpha}
$$

we obtain

$$
p^{\prime}(z) F^{n-1}(z)+\frac{p^{\prime \prime}(z)}{2} F^{n-2}(z)+\cdots+\frac{1}{n !} p^{(n)}(z)=\frac{s(z)}{Q(z)^{n}} e^{\gamma(z)-n \alpha}
$$

or

$$
F^{n-1}(z)+\frac{p^{\prime \prime}(z)}{2 p^{\prime}(z)} F^{n-2}(z)+\cdots+\frac{p^{(n)}(z)}{n ! p^{\prime}(z)}=R(z) e^{\gamma-n \alpha},
$$

where $R(z)=s(z) / Q^{n}(z) p^{\prime}(z)$, a rational function.

The- left-hand side of (8) is a polynomial in $F$ with rational functions as its coefficients. Thus, Lemma 1 is applicable unless $F$ is a rational function.

Suppose $F$ is a rational function. Then from (6), it follows that

$$
f_{2}(p)=Q(z) R_{1}(z) e^{\alpha},
$$

where $R_{1}(z)$ is a rational function.

Then from (2), we have

$$
f_{1}(p)=R_{2}(z) e^{\alpha(z)},
$$

where $R_{2}(z)$ is a rational function.

Thus, we have $f_{1}(p) / f_{2}(p)=R_{2} / Q R_{1}=$ rational function, which of course, is impossible.

Alternately, we conclude that $F$ is transcendental. We note that $T$ has only finitely many poles, thus applying Lemma 1 to the identity (8) and noting remark (i), of Lemma 1, we have

$$
\left[F(z)+\frac{1}{n-1} \cdot \frac{p^{\prime \prime}(z)}{2 p^{\prime}(z)}\right]^{n-1}=R(z) e^{\gamma-n \alpha} .
$$


Combining this with (8), we obtain

$$
\left[F(z)+\frac{1}{n-1} \cdot \frac{p^{\prime \prime}(z)}{2 p^{\prime}(z)}\right]^{n-1} \equiv F^{n-1}(z)+\frac{p^{\prime \prime}(z)}{2 p^{\prime}(z)} F^{n-2}(z)+\cdots+\frac{p^{n}(z)}{n ! p^{\prime}(z)} .
$$

By remark (ii) of Lemma 1, equating the coefficients of both sides of (11), we obtain

$$
\left[\frac{1}{2(n-1)} \cdot \frac{p^{\prime \prime}(z)}{p^{\prime}(z)}\right]^{n-1}=\frac{p^{(n)}(z)}{n ! p^{\prime}(z)}
$$

or

$$
\left[p^{\prime \prime}(z)\right]^{n-1}=\frac{d(2(n-1))^{n-1}}{n !} \cdot\left[p^{\prime}(z)\right]^{n-2},
$$

where $d=p^{(n)}(z)$ is a constant.

Hence, by a simple degree argument, $p^{\prime}(z)=c(z-a)^{n-1}$, where $c$ is a constant.

It follows that $p(z) \bar{z} c_{1}(z-a)^{n}+c_{2}$ for some constants $c_{1}, c_{2}$.

Substituting in equations (3) and dividing through both sides of (3) by $f_{2}^{n}(z)$, we get

$$
c_{1}(f(z)-a)^{n}+c_{2}-z=\left(R_{0}(z) / f_{2}^{n}(z)\right) e^{\beta(z)} .
$$

Let us change variables in (13), by setting $z=c_{2}+c_{1} w^{n}$, and $f(z)-a=H(w)$. We have

$$
c_{1}\left(H^{n}(w)-w^{n}\right)=\left(R\left(c_{2}+c_{1} w^{n}\right) / f_{2}\left(c_{2}+c_{1} w^{n}\right)\right) \exp \left(\beta\left(c_{2}+c_{1} w^{n}\right)\right) .
$$

When $n \geqq 3$, (14) implies that

$$
H(w) / w-\rho_{i}=0
$$

$\left(\rho_{1}, \rho_{2}, \rho_{i}, \ldots, \rho_{n}\right.$ are $n$ distinct roots of unity) has only finitely many zeros, for $i=1,2,3, \ldots, n$.

According to Nevanlinna's second fundamental theorem, or Picard's theorem we conclude at once that $H(w)$ must be a rational function. This contradicts our hypothesis that $f(z)$ is transcendental. The theorem is thus proved.

Proof of Theorem 3. Let $f(z)=f_{1}(z) / f_{2}(z)$. Suppose that $(f(z))^{2}$ has only finitely many fix-points. Then we have

$$
f_{1}^{2}(z)-z f_{2}^{2}(z)=Q_{0}(z) e^{\alpha(z)},
$$

where $Q_{0}(z)$ is a polynomial and $\alpha(z)$ is an entire function.

Replacing $z$ by $z^{2}$ in the above identity, we have

$$
f_{1}^{2}\left(z^{2}\right)-z^{2} f_{2}^{2}\left(z^{2}\right)=Q_{0}\left(z^{2}\right) \exp \left(\alpha\left(z^{2}\right)\right)
$$

or

$$
\left[f_{1}\left(z^{2}\right)+z f_{2}\left(z^{2}\right)\right]\left[f_{1}\left(z^{2}\right)-z f_{2}\left(z^{2}\right)\right]=Q_{0}\left(z^{2}\right) \exp \left(\alpha\left(z^{2}\right)\right) .
$$


Hence, we conclude

$$
f_{1}\left(z^{2}\right)+z f_{2}\left(z^{2}\right)=Q(z) e^{\gamma(z)}
$$

Change $z$ into $-z$ to obtain

$$
f_{1}\left(z^{2}\right)-z f_{2}\left(z^{2}\right)=Q(-z) e^{\gamma(-z)},
$$

where $Q \dot{(z)}$ is a polynomial, and $\gamma$ is an entire function.

Therefore

$$
f_{1}(z)=\frac{1}{2}\left(Q\left(z^{1 / 2}\right) \exp \left(\gamma(z)^{1 / 2}\right)+Q\left(-z^{1 / 2}\right) \exp \left(\gamma(-z)^{1 / 2}\right)\right),
$$

and

$$
f_{2}(z)=\left(1 / 2 z^{1 / 2}\right)\left(Q\left(z^{1 / 2}\right) \exp \left(\gamma\left(z^{1 / 2}\right)\right)-Q\left(-z^{1 / 2}\right) \exp \left(\gamma\left(-z^{1 / 2}\right)\right)\right) .
$$

Finally, we observe that if one chooses $f(z)=f_{1}(z) / f_{2}(z)$, with

and

$$
f_{1}(z)=\frac{1}{2}\left(Q\left(z^{1 / 2}\right) \exp \left(\gamma\left(z^{1 / 2}\right)\right)+Q\left(-z^{1 / 2}\right) \exp \left(\gamma\left(-z^{1 / 2}\right)\right)\right),
$$

$$
f_{2}(z)=\left(1 / 2 z^{1 / 2}\right)\left(Q\left(z^{1 / 2}\right) \exp \left(\gamma\left(z^{1 / 2}\right)\right)-Q\left(-z^{1 / 2}\right) \exp \left(\gamma\left(-z^{1 / 2}\right)\right)\right)
$$

then $f\left(z^{2}\right)$ has only finitely many fix-points. Hence, $(f(z))^{2}$ also has only finitely many fix-points. The theorem is thus proved.

Proof of Theorem 1. Suppose that $F=f(g)$ has only finitely many fix-points. If one of $f$ or $g$ is a polynomial, then Theorem B together with the observation in the beginning of the proof of Theorem 2 yields the assertion. Now, suppose both $f$ and $g$ are transcendental, and $f(g)$ has only finitely many fix-points. This yields $f(g)-z=P(z) e^{Q(z)}$, where $P, Q$ are polynomials. Hence, $P(z) e^{Q(z)}+z=f(g)$. This is impossible according to Lemma 2, and the theorem follows.

Proof of Theorem 4. Suppose that the assertion is false. By virtue of Lemma 3, it suffices to show that $h(z)$ is $E$-prime. Assume that there exists a pair of nonlinear entire functions $f$ and $g$ such that

$$
f(g)=q(z) \exp \left(a z^{2}+b z\right)+p(z) .
$$

According to Lemma 2, either $f$ or $g$ must be a polynomial.

Suppose that $f$ is a polynomial, $Q_{1}$, say, and suppose that $g$ is transcendental entire.

Then we have

$$
Q_{1}(g)-p(z)=q(z) \exp \left(a z^{2}+b z\right)
$$

where $Q_{1}(g)$ is a polynomial of degree $\geqq 2$. Applying Lemma 1 , one easily arrives at a contradiction. Thus, we need only show that (20) cannot hold when $g$ is a polynomial and $f$ is transcendental entire. Since the right-hand side of (20) has infinitely many zeros (see e.g. Nevanlinna [11, p. 76]), $f$ must have infinitely many zeros. We may assume without loss of generality that $a=1$ and $b=0$ (if $a=0$, we assume 
$\operatorname{Re} b>0)$. Then it is easy to verify that the zeros of $q(z) \exp \left(z^{2}\right)+p(z)$ will be asymptotic to two half rays, say $R_{1}$ and $R_{2}$. Now if the degree of $g$ is greater than 2 , then one can find a sequence of zeros $a_{i}$ of $f$ and a corresponding sequence of zeros $\rho_{i}$ of $g(z)-a_{i}$ such that the sequence $\rho_{i}$ is not asymptotic to either $R_{1}$ or $R_{2}$, a contradiction.

To verify the latter statement simply note that whenever $q$ and $p$ are of different degrees, the equation (21) implies either $\exp \left(z^{2}\right) \rightarrow 0$ or $\exp \left(z^{2}\right) \rightarrow \infty$, i.e. either $\operatorname{Re} z^{2}<0$ or $\operatorname{Re} z^{2}>0$. The case where the degrees of $q$ and $p$ are the same can be reduced to the above by differentiating (21).

Thus we conclude that the degree of $g$ is two and we may assume that $g$ has the form $g(z)=(z+c)^{2}+d$.

Then

$$
f\left((z+c)^{2}+d\right)=q(z) \exp \left(a z^{2}\right)+p(z) .
$$

Hence

$$
q(z) \exp \left(a z^{2}\right)+p(z)=q(-z-2 c) \exp \left(a(-z-2 c)^{2}\right)+p(-z-2 c) .
$$

By virtue of Lemma 4 , this is possible only when $p(z)=p(-z-2 c), b=2 a c$, $q(-z-2 c)=q(z)$, contrary to our hypotheses. Theorem 4 is thus proved.

Corollary of Theorem 4 immediately follows since Lemma 4 implies that $g$ cannot be of second degree.

In conclusion, we note that by using Lemma 2 , one can verify that if $f(z)$ is a transcendental meromorphic function with only finitely many poles, then $R(f)$ has infinitely many fix-points, where $R(z)$ is a rational function but not of bilinear form. More generally, we conjecture that if $R(z)$ is any rational function of weight greater than 2 and $f$ is transcendental meromorphic, then $R(f)$ has infinitely many fix-points. However, it can be shown by Lemma 2 and Picard's theorem that when $f$ is a meromorphic function with its number of poles satisfying $N(r, f)=S(r, f)$, then for any rational function $R(z)$ of weight greater than $2, R(f)$ must have infinitely many fix-points.

REMARK. Using a different argument, the present authors have been able to obtain some generalizations of Theorems 4, 5 and 6 . These results will be published in a subsequent paper.

\section{REFERENCES}

1. N. Baker and F. Gross, Further results on factorization of entire functions, Proc. Sympos. Pure Math., vol. 11, Amer. Math. Soc., Providence, R. I., 1969, pp. 30-35.

2. E. Borel, Sur les zéros des fonctions entières, Acta Math. 20 (1897).

3. A. Edrei and W. H. J. Fuchs, Valeurs déficientes et valeurs asymptotiques des fonctions méromorphes, Comment. Math. Helv. 33 (1959), 258-295. MR 23 \#A1038.

4. P. Fatou, Sur litération des fonctions transcendantes entières, Acta Math. 47 (1926), 337-370.

5. F. Gross, Factorization of meromorphic functions, U.S. Government Printing Office, Washington, D. C. (to appear). 
6. F. Gross, On factorization of meromorphic functions, Trans. Amer. Math. Soc. 131 (1968), 215-222. MR 36 \#3988.

7. - Factorization of entire functions which are periodic $\bmod g$, Indian J. Pure Appl. Math. 2 (1971), 561-571.

8. __ - Prime entire functions, Trans. Amer. Math. Soc. 161 (1971), 219-233.

9. W. K. Hayman, Meromorphic functions, Oxford Math. Monographs, Clarendon Press, Oxford, 1964. MR 29 \#1337.

10. R. Goldstein, On factorization of certain entire functions, J. London Math. Soc. (2) 2 (1970), 221-224. MR 41 \#2012.

11. R. Nevanlinna, Le théorème de Picard-Borel et la théorie des fonctions méromorphes, Paris, 1929.

12. P. C. Rosenbloom, The fix-points of entire functions, Medd. Lunds Univ. Mat. Sem Tome Supplémentaire 1952, 186-192. MR 14, 546.

Department of Mathematics, University of Maryland, Baltimore, Maryland 21228 (Current address of first author)

Mathematics Research Center, Naval Research Laboratory, Washington, D.C. 20390 (Current address of both authors) 